Riklef Weerda $\cdot$ Ignacio Vallines $\cdot$ James P. Thomas

Roland M. Rutschmann • Mark W. Greenlee

\title{
Effects of nonspatial selective and divided visual attention on fMRI BOLD responses
}

Received: 8 April 2005/ Accepted: 26 January 2006/Published online: 9 March 2006

(C) Springer-Verlag 2006

\begin{abstract}
Using an uncertainty paradigm and functional magnetic resonance imaging (fMRI) we studied the effect of nonspatial selective and divided visual attention on the activity of specific areas of human extrastriate visual cortex. The stimuli were single ovals that differed from an implicit standard oval in either colour or width. The subjects' task was to classify the current stimulus as one of two possible alternatives per stimulus dimension. Three different experimental conditions were conducted: "colour-certainty", "shape-certainty" and "uncertainty". In all experimental conditions, the stimulus differed in only one stimulus dimension per trial. In the two certainty conditions, the subjects knew in advance which dimension this would be. During the uncertainty condition they had no such previous knowledge and had to monitor both dimensions simultaneously. Statistical analysis of the fMRI data (with SPM2) revealed a modest effect of the attended stimulus dimension on the neural activity in colour sensitive area V4 (more activity during attention to colour) and in shape sensitive area LOC (more activity during attention to shape). Furthermore, cortical areas known to be related to attention and working memory processes (e.g., lateral prefrontal and posterior parietal cortex) exhibit higher activity during the condition of divided attention ("uncer-
\end{abstract}

\footnotetext{
R. Weerda $(\bowtie)$

Section of Cognitive Research, Department of Psychology, School of Humanities and Social Sciences, Carl von Ossietzky University Oldenburg, 26111, Oldenburg, Germany

E-mail: Riklef.Weerda@GMX.de

Tel: $+49-441-7985154$

Fax: + 49-441-7985170

I. Vallines · R. M. Rutschmann · M. W. Greenlee Department of Experimental Psychology, University of Regensburg, Regensburg, Germany

J. P. Thomas

Department of Psychology, University of California, Los Angeles, CA, USA

J. P. Thomas

Hanse Institute for Advanced Study, Delmenhorst, Germany
}

tainty") than during that of selective attention ("certainty").

Keywords Stimulus uncertainty - Visual attention · Colour discrimination - Shape perception - Functional MRI

\section{Introduction}

Human visual cortex contains various areas that are specialised in the processing of certain aspects of visual information, like colour sensitive area V4 (e.g., Bartels and Zeki 2000) or shape sensitive area LOC (lateral occipital complex) (Malach et al. 1995; Kourtzi and Kanwisher 2000, 2001; Grill-Spector et al. 2001; Lerner et al. 2001). During each fixation period, countless bits of visual information fall on the retina, exceeding that which can be processed extensively by the brain. To select among these different sources of information, we rely on attention to direct our processing resources to behaviourally relevant features of visual scenes. Among these attentional selection mechanisms are selective and divided attention: during selective attention, only one channel of information is attended, whereas during divided attention, at least two different channels are monitored simultaneously. In the special case of nonspatial attention, the different features that can be attended to are that of one single object, so that spatial position is not one of the critical features and attention is always allocated to the same region within the visual field.

There is accumulating evidence suggesting that the exact pattern of cortical activation varies depending on which features subjects selectively attend to during a discrimination task. For example, Huk and Heeger (2000) found increased activity in motion sensitive area MT + when subjects had to perform speed discriminations compared to when they had to discriminate different contrasts. In a positron emission tomography (PET) study, Corbetta et al. (1990, 1991) showed that 
dependent on the attended stimulus feature (colour, shape or speed) different extrastriate visual areas become activated and that dependent on the kind of attentional mechanism (selective or divided) different parts of the frontal lobes and the basal ganglia are active. One problem in that study is that while the subjects were instructed to attend to subtle changes in one stimulus feature, the other two features could change as well and it remains controversial as to what extent the subjects ignored these irrelevant stimulus changes. Le et al. (1998) measured blood oxygenation level dependent (BOLD) responses during sustained or alternately shifted attention to the colour or shape of foveally presented stimuli. They found evidence for feature-specific activations in occipital and temporal visual areas and greater activity in the posterior superior parietal lobule, cuneus, precuneus and different parts of the cerebellum during shifts of attention than during sustained attention. However, they measured cortical activity only in the posterior half of the brain, so that nothing could be inferred about the involvement of prefrontal areas in the attentional control system. With respect to the investigation of the effects of attentional selection on featurespecific activations, the use of large stimulus differences (red vs. green, circle vs. square) is problematic, since several psychophysical studies showed larger attentional effects for near-threshold discriminations (e.g., Vogels et al. 1988; Blanco and Soto 2002). Furthermore, the large stimulus differences by themselves might, at least partially, explain differential cortical activations.

Simultaneous discrimination of stimuli that can differ along one or another dimension has been used in psychophysics to explore the independence of visual processing, for example in experiments concerning visual dimension weighting (e.g., Weidner et al. 2002). One paradigm used to investigate this question has been referred to as stimulus uncertainty (Tanner 1956; Sperling 1986). For the case of the colour and shape of a given object, it has been shown that judgements about variations can be made independently when the differences are large and the observer has prior information about which dimension should be judged (see Treisman 1986). What happens when the differences to be discriminated are small and the observer is uncertain about which dimension will change? In the stimulus uncertainty paradigm, observers have to allocate their attentional resources across at least two different stimulus features. Owing to the effects of internal noise, uncertainty has been shown to increase discrimination thresholds in detection and discrimination tasks (Thomas et al. 2000; Magnussen and Greenlee 1997; Thomas and Olzak 1996; Graham 1989; Pelli 1985).

In our study, we employed an uncertainty paradigm and functional magnetic resonance imaging (fMRI) in order to examine whether there are differences in cortical activity that are solely attributable to differential shifts of nonspatial selective visual attention to selected features of an object. More precisely, we looked for changes in the BOLD signal of specialised areas of human extrastriate visual cortex that are known to be more sensitive either to the colour or shape of objects. If there are differences, then the signal should be stronger when the respective stimulus feature is attended to. Additionally, we explored if there are any differences in brain activity between selective and divided attention, presumably in areas belonging to the so-called "frontoparietal attention network" that are known to be related to attentional and working memory processes, like posterior parietal and lateral prefrontal cortex. We expect the neural activity to be higher during divided attention, since here the demand on attentional and working memory processes is higher than during selective attention.

\section{Methods}

\section{Subjects}

Four female and two male students, aged 24 to 26 years, participated in this study after providing written informed consent. All of them are right-handed and have normal or corrected-to-normal (with contact lenses) visual acuity. Their colour vision was tested before the experiment with test plates no. 1-25 of "Ishihara's tests for colour-blindness" (Ishihara 1982) and all subjects gave 100\% correct answers, indicating normal colour vision.

\section{Stimuli and task paradigms}

The stimulus was an oval of fixed height $\left(4.38^{\circ}\right.$ visual angle) and luminance $\left(50 \mathrm{~cd} / \mathrm{m}^{2}\right)$. In each trial, the presented oval differed slightly from an implicit, but never shown, standard oval in either colour (hue) or shape (width) by an individually adjusted fixed amount. See Morgan et al. (2000) for the general usefulness of an implicit standard instead of an explicitly presented reference stimulus in visual discrimination experiments. We decided to use an implicit standard since this allows us to just present the target stimulus that has to be judged by the subject and to measure the BOLD response to this stimulus without possible interferences with the response to an explicitly shown reference stimulus.

The stimulus colour was specified in CIE $x y Y$ coordinates (CIE Proceedings 1932). The implicit standard was pink in appearance with the coordinates $0.43,0.224$, 50. The two different alternatives were either bluer or redder, where the extent of the difference between these two alternatives was individually adjusted in order to make the difficulty level comparable for each subject. The individual differences ranged from 0.017 to 0.059 in the $x$ value and from 0.008 to 0.028 in the $y$ value. The shape of the standard oval was set at a height of $4.38^{\circ}$ and a width of $3.09^{\circ}$ visual angle. Increasing or decreasing the width of the oval by a small amount specified the shape of the actual stimulus presented to the subject. The extent of the difference between the two 
alternatives was again individually adjusted and ranged from $0.09^{\circ}$ to $0.26^{\circ}$. Figure 1 shows exaggerated examples of the two possible alternatives per stimulus dimension. Each stimulus was presented against a dark background for $250 \mathrm{~ms}$. The dark background was $13.13^{\circ}$ wide by $8.75^{\circ}$ high and framed by a thin (width $\left.=2.19^{\circ}\right)$, white $(x y Y=0.27,0.28,75)$ border.

The task of the observer was to classify the actual stimulus as one of two alternatives per stimulus dimension. There were three different experimental conditions: Two certainty conditions (one for colour and one for shape), in which the subjects knew on which dimension the deviation would occur, and one uncertainty condition, in which colour and shape deviations were randomly intermixed from trial to trial, so that the subjects had no previous knowledge about the varied stimulus feature. The stimulus differences were small enough that accuracy was less than perfect, i.e. discrimination performance was noise limited.

In the colour-certainty condition, the subjects indicated whether the stimulus was bluer $($ response $=1$ ) or redder (response $=2$ ) than the standard, while in the shape-certainty condition, the subjects indicated whether the stimulus was narrower (response $=1$ ) or wider (response $=2$ ). In the uncertainty condition, the subjects again gave a single response on each trial: he or she pressed button 1 if the stimulus was judged bluer or narrower than the standard, button 2 if the stimulus was judged redder or wider. Note that the relationships between stimulus and response are the same in certainty and uncertainty conditions. We decided to employ this singlestimulus single-response procedure with two response alternatives, since it was best suited for the application in the MR scanner. Because of the spatial circumstances in the MR scanner and the technical requirements demanding rapid trial sequences, the subjects only responded with two fingers of one hand. The response interval ( $1 \mathrm{~s})$ was adjusted to require a speeded response.

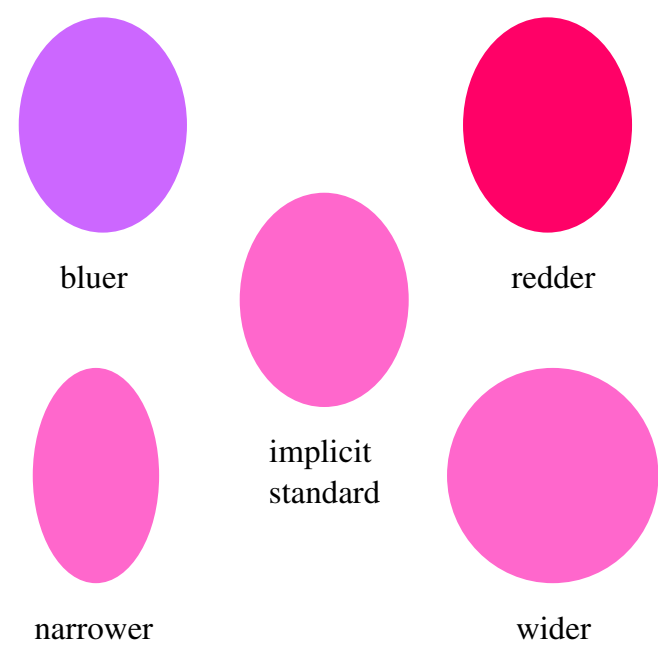

Fig. 1 The implicit standard stimulus and exaggerated examples of the four different stimulus alternatives. One alternative was presented centrally on each trial
In all conditions, a central white fixation point was displayed between stimulus presentations. After each response, feedback indicating whether the response was correct or incorrect was given via a brief $(250 \mathrm{~ms})$ colour change of the fixation point (green $=$ correct, red $=$ incorrect). This feedback was given in order to keep the subjects' performance at the previously trained level and to assure that they generally performed on their best achievable level and thereby concentrated on the respective stimulus feature that had to be monitored. The trial-to-trial interval was $2 \mathrm{~s}$. Figure 2 shows a schematic illustration of the experiment's time course.

Before the main experiment, each subject was run in several sessions in the psychophysics laboratory. These sessions were part of the study by Vallines et al. (2001) and accomplished two goals: (1) to select individual deviation values (between stimulus alternatives) by measuring the psychometric functions, relating deviation magnitude to percentage of correct answers, for each subject in order to adjust the difficulty level so that it was the same for all subjects; (2) to train the subjects in the tasks that they would perform in the MR scanner. The individual deviation values were achieved by letting the subjects perform the task under the two certainty conditions while varying the extent of the differences between the two stimulus alternatives for each dimension (colour and shape). Then the observed performance data for the various difference values (deltas) were fitted with the Weibull model and for each subject and each stimulus dimension a certain delta value was chosen such that it yielded a performance level of about $90 \%$ correct answers.

\section{Procedure}

The subjects lay supine within the MR scanner. A mirror was mounted above the head coil through which they looked out of the foot end of the scanner bore, where a rear projection screen was placed on which the stimuli were presented with an LCD projector (D-ILA Projector, DLA-G10; JVC, Yokohama, Japan). The RGB voltage-luminance functions of the projector were calibrated with a spectral photometer. The stimuli were generated with a VSG graphics card (Cambridge Research Systems Ltd., Rochester, UK), which was also used for response acquisition. The subjects responded as described above with an optical response box (Cambridge Research Systems Ltd.). This response box is made entirely of plastic materials and a fibre-optic cable in order to avoid artefacts caused by current flows within the magnetic field. It was placed on the right hand for each subject.

Measurements within the scanner were blocked into six runs (two per experimental condition), each consisting of nine successive 1-min segments (block-design). Five of the segments were rest conditions in which the stimulus field was blank except for a fixation point at the centre. Alternating with the rest segments were 
Fig. 2 Schematic illustration of the experiment's time course

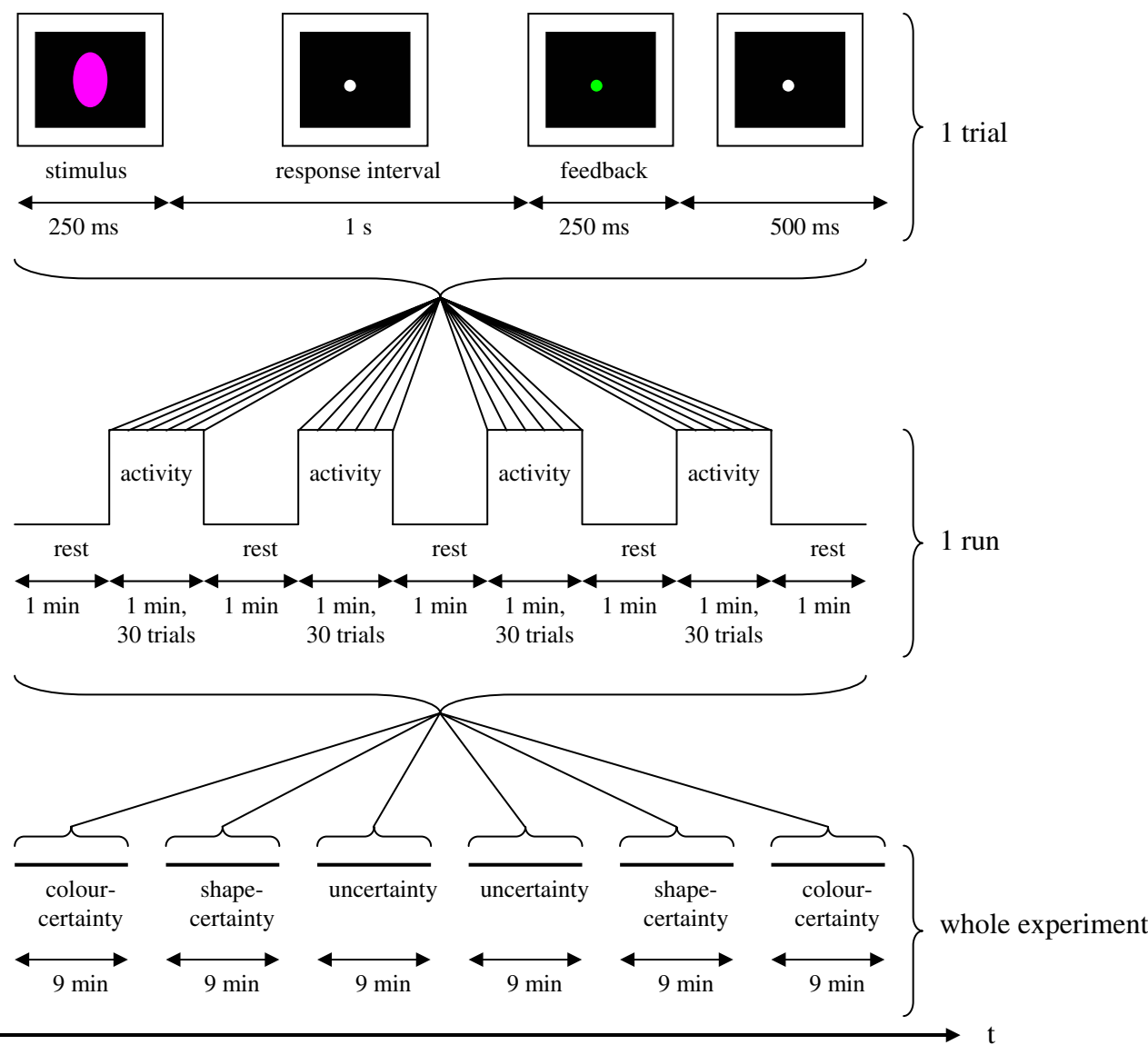

judgement segments in which one stimulus was presented every $2 \mathrm{~s}$. The experimental condition remained the same within each run and right before the beginning of a new run, the subject was told verbally which condition would come next. The order of the different conditions was colour-certainty, shape-certainty, uncertainty, uncertainty, shape-certainty and colour-certainty (A-B-C-C-B-A) (see Fig. 2).

\section{MRI data acquisition}

The MR scanner used for this experiment is a 1.5 Tesla whole body Siemens Vision Magnetom. Every $6 \mathrm{~s}$ $[=$ time to repeat $(\mathrm{TR})]$ one functional whole-brain $\mathrm{T}_{2}{ }^{*}$-weighted gradient echo planar imaging (EPI) scan was carried out (time to echo (TE) $=60 \mathrm{~ms}$; flip angle $\alpha=90^{\circ}$ ), each consisting of 32 contiguous slices (no gap) parallel to the anterior commissure-posterior commissure (AC-PC) plane with each slice being $64 \times 64$ voxels and a voxel size of $3 \times 3 \times 3 \mathrm{~mm}$. Following the six experimental runs, an anatomical $\mathrm{T}_{1}$-weighted scan (160 slices, each slice $512 \times 512$ voxels) was obtained in sagittal orientation, using a magnetisation prepared rapid acquisition gradient echo (MPRAGE) sequence with $\mathrm{TR}=9.7 \mathrm{~ms}, \mathrm{TE}=4 \mathrm{~ms}$, inversion time $=300 \mathrm{~ms}$ and $\mathrm{a}$ voxel size of $1 \times 0.5 \times 0.5 \mathrm{~mm}$.

\section{Data analysis}

\section{Psychophysical data analysis}

Accuracy was measured by percentage of correct responses and $\mathrm{d}^{\prime}$. In signal detection theory, $\mathrm{d}^{\prime}$ is a measure of the sensitivity of a subject for the discrimination of two different stimuli on a feature continuum. It can be computed based on the hit and false alarm rates of the subject. For our purposes, stimuli which were bluer or narrower than the standard were arbitrarily designated noise and redder or wider stimuli were designated signal, and responses of 1 and 2 were treated as "no" and "yes", respectively (Wickens 2001). Response times, measured from the onset of the stimulus to activation of the response button, were also recorded. On a few trials (less than $1 \%$ ), the subjects did not respond within the excluded from the calculations.

\section{fMRI data analysis}

The functional imaging data were analysed using SPM2 (Statistical Parametric Mapping 2002), a computer programme running under Matlab (see http://www.fil.ion.ucl.ac.uk/spm/ for further details). All images were realigned and unwarped in order to 1-s time window allowed. Data from those trials were 
eliminate motion artefacts and spatially normalised to the standard stereotactic space of Talairach and Tournoux (1988) with a bilinear interpolation method. Smoothing was performed by a three-dimensional convolution of the functional images with an isotropic Gaussian kernel of $8 \mathrm{~mm}$ full width at half maximum, which accomplished the goals of increasing the signal to noise ratio and minimising the effects of individual differences in functional and gyral anatomy. Afterwards, an appropriate design matrix was specified for the pooled data of all subjects (fixed effects analysis). Changes in the BOLD signal dependent on the different experimental conditions were assessed at each voxel by using a general linear model with a boxcar waveform convolved with the haemodynamic response function and by applying the theory of Gaussian fields (Friston et al. 1995). To make statistical inferences about regionally specific effects (i.e., assign $p$-values), the estimates were compared using linear contrasts. $t$-values were assigned to every voxel and the resulting set of voxel values for each contrast constituted a statistical parametric map (SPM) of the $t$-statistic, $\operatorname{SPM}(\mathrm{t})$, which was then thresholded at $p \leq 0.05$ (corrected for multiple comparisons by means of the false discovery rate). Additional to the whole brain analysis, Regions of Interest (ROIs) were defined [V4, V4 $\alpha$, LOC, posterior parietal cortex [Brodmann Areas (BA) 7 and 40] and prefrontal cortex (BAs 44, 45, 46, 47, 9 and 10)] based on normalised coordinates available in the literature (Bartels et al. 2000, for area V4 and V4 $\alpha$; Malach et al. 1995, and Lerner et al. 2001, for area LOC). The Talairach coordinates for area V4 ranged from \pm 20 to $\pm 38(x),-65$ to $-85(y)$ and -4 to $-24(z)$, for area $\mathrm{V} 4 \alpha$ from \pm 24 to $\pm 38(x),-44$ to $-62(y)$ and -5 to $-24(z)$ and for area LOC from \pm 32 to $\pm 50(x),-52$ to $-86(y)$ and -28 to $10(z)$. By applying the method of ROI analysis, the statistical threshold is decreased for voxels within this ROI, due to the smaller absolute number of analysed voxels and the correspondingly reduced correction for multiple comparisons.

\section{Results}

\section{Psychophysical results}

Table 1 presents performance values for each subject and experimental condition. In this forced-choice task, chance performance corresponds to $50 \%$, and the onetailed $95 \%$ confidence limit for the uncertainty condition lies at $58 \%$ correct answers. The table also shows individual $\mathrm{d}^{\prime}$ values and reaction times for the six subjects. The different trials in the uncertainty condition were scored separately as colour- and shape-uncertainty trials. The magnitudes of the individual uncertainty effects are given as ratios of $\mathrm{d}^{\prime}$ in the uncertainty condition to $\mathrm{d}^{\prime}$ in the corresponding certainty condition. Smaller ratios indicate larger uncertainty effects.
As can be seen in Table 1, performance in the uncertainty condition is lower than the corresponding performance in the certainty condition. An analysis of variance (ANOVA) on the $\mathrm{d}^{\prime}$ values shows a main effect of certainty-uncertainty $[F(1,5)=18.383, p<0.01]$. Consistent with the accuracy results, reaction times were longer in the uncertainty than in the certainty conditions $[F(1,5)=8.951, p=0.03]$.

Although the stimulus differences to be discriminated were individually adjusted to make colour and shape discriminations equally accurate, the actual scores of three of the subjects (IV, JM, MS) are markedly lower on the colour task than on the shape task. The lower performance occurs in both certainty and uncertainty conditions and, in the latter case, none of the scores for these three subjects exceeds the $95 \%$ confidence limit for chance performance. As measured by the ratios in Table 1, the uncertainty effects for these subjects in the colour task are much larger than the effects observed for these same subjects in the shape task and larger than any of the uncertainty effects observed for the other three subjects. The reasons for these performance differences of these three subjects between the data collected in the psychophysics laboratory and in the MR scanner remain unclear.

Functional imaging results

\section{Contrasts against rest}

Because of pronounced differences in stimulation between the rest conditions and the experimental conditions, several sites of significant activation are evident in the contrasts with rest. The ROI analysis of the contrast between the colour-certainty and the rest condition revealed more activity in areas V4 and $\mathrm{V} 4 \alpha$ as well as in area LOC. The whole brain analysis of the same contrast showed additional activation differences in posterior parietal (BAs 7 and 40), anterior cingulate (BA 32) and prefrontal cortex (BAs 9, 44, 47), as well as in the frontal eye fields.

The ROI analysis of the contrast between the shapecertainty and the rest condition also resulted in significantly more activity in areas $\mathrm{V} 4, \mathrm{~V} 4 \alpha$ and LOC, whereas a whole brain analysis revealed results in posterior parietal (BA 40), anterior cingulate (BA 32) and prefrontal cortex (BAs 9, 44, 46, 47) and the frontal eye fields.

In order to see if there were any differences in the examined ROIs between these two contrasts, we masked them against one another. When the contrast "colour-certainty $>$ rest" is masked with the contrast "shape-certainty > rest", there are remaining activity differences in areas V4 and V4 $\alpha$ in the left hemisphere (see Fig. 3a), but not in area LOC. The reverse masking ended up in more mixed results: remaining activity in area LOC (see Fig. 3c), but also in area V4 in the right hemisphere. 
The ROI analyses of the contrast between the conditions of uncertainty and rest established more activity in all previously described ROIs (V4, V4 $\alpha$, LOC, posterior parietal (BAs 7 and 40) and prefrontal cortex (BAs $9,10,46)$ ), whereas whole brain analysis revealed additional activity in the anterior cingulate cortex (BA 32), the frontal eye fields and premotor BA 6. By masking this contrast with the two contrasts between the two certainty conditions and the rest condition, we found remaining activity differences in all the above-mentioned areas with the exception of area V4 and the frontal eye fields.

\section{Differential contrasts}

In order to test the statistical significance of the differences in activity found by the above-mentioned masking of different contrasts against rest with each other, direct comparisons between the different experimental conditions were calculated. The ROI analysis of the contrast "colour-certainty > shape-certainty" revealed only a very small activity difference in area V4 (see Fig. 3b) in the left hemisphere. Additional differences in activation found in the whole brain analysis of the same contrast lie at the border between BAs 10 and 46 in the right hemisphere and in BA 32 on the anterior cingulate gyrus.

The ROI analysis of the reverse contrast gave no significant differences in cortical activity in the LOC. The whole brain analysis of the same contrast resulted in significant differences in a medial-orbitofrontal area at the border between BAs 10 and 11, in the posterior parietal cortex (precuneus) and in BA 21 on the middle temporal gyrus.

The direct comparison between the uncertainty condition on the one hand and the two certainty conditions on the other hand resulted in considerably higher activity in posterior parietal cortex (BAs 7 and 40, see Fig. 4a), premotor BA 6 and in prefrontal cortex (BAs 9, 10, 46; see Fig. 4b) during uncertainty.

Additional to the group analysis, all of the abovementioned differential contrasts have also been calculated separately for each subject in order to test if the large interindividual differences in performance could have cancelled out possible effects. None of the subjects showed any differences in cortical activity in $\mathrm{V} 4, \mathrm{~V} 4 \alpha$ or LOC, but in all of them more activity during uncertainty than during certainty was evident in the posterior parietal and prefrontal cortex. Due to the limited number of subjects in the study, a correlation between performance and BOLD response in the ROIs was not determined.

\section{Discussion}

An uncertainty paradigm and fMRI were used to examine the effects of nonspatial selective and divided visual attention on the sensitivity to near-threshold 
Fig. 3 Regions of interest (ROI) analysis of extrastriate visual areas. a Area V4 in the contrast colour-certainty $>$ rest b Area V4 in the contrast colour-certainty $>$ shapecertainty. c Area LOC in the contrast shape-certainty $>$ rest. Sagittal, coronal and axial overlays of pooled functional data of all subjects and a normalised mean anatomical image. Colours code t-statistic. Left in the image corresponds to left in the brain a) colour-certainty $>$ rest
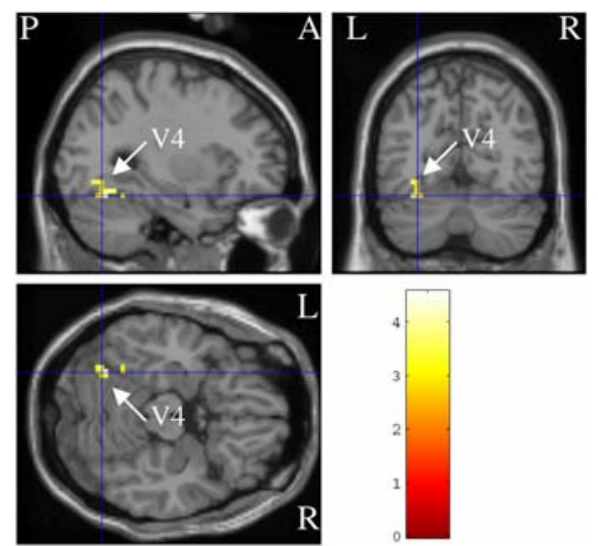

c) shape-certainty $>$ rest
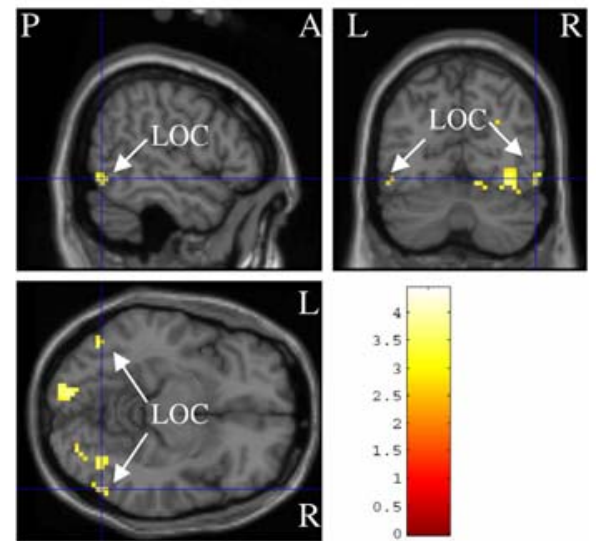

b) colour-certainty $>$ shape-certainty
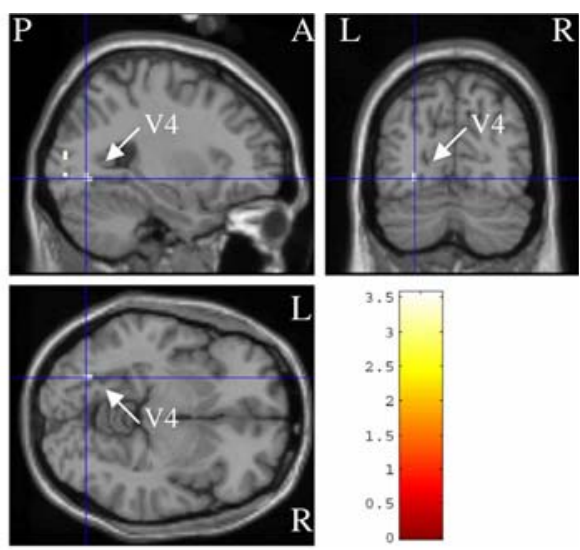

differences in colour or shape, and on cortical activation patterns evoked by perceptually nearly identical stimuli.

We explored whether there are differences in cortical activity in the specialised areas V4 and LOC of human extrastriate visual cortex between selective attention to subtle changes in the colour of an object and attention to subtle changes in its shape, which are only attributable to the differential shift of attention.
Furthermore, we examined what happens when subjects have to attend to two different stimulus features simultaneously. Are the same cortical areas active as during selective attention to these stimulus features or are there differences in cortical activity that are dependent on the kind of attentional mechanism, presumably in cortical areas known to be related to attentional and working memory processes?
Fig. 4 ROI analysis of the contrast uncertainty $>$ certainty: a Posterior parietal BAs 7 and 40. b Lateral prefrontal BA 46. In the lower right corner, so-called "glass brains" are displayed with the Talairach grid overlay and greyscale-coded $T$-values. Each glass brain presents $2 \mathrm{D}$ projections of the activations onto the standard brain; otherwise as in fig. 3 a)

$$
\text { posterior parietal cortex }
$$

$$
\text { (BAs } 7 \text { and 40) }
$$
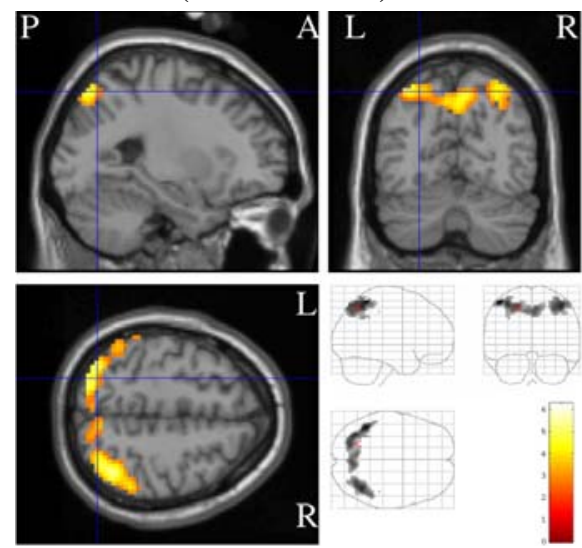

b)

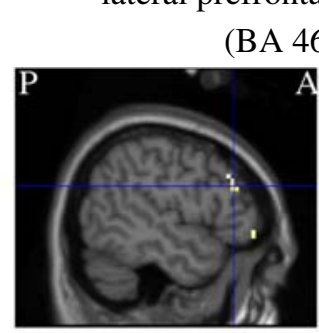

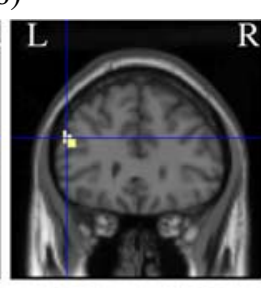
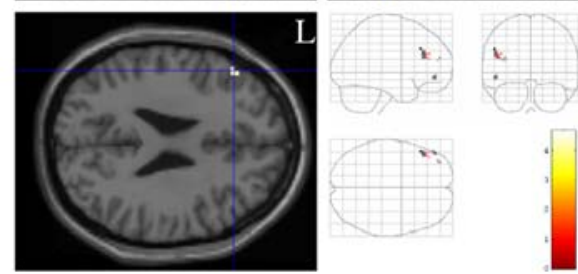
The present results point towards a modest effect of the attended stimulus feature on the neuronal activity in specialised areas of human extrastriate visual cortex. The method of masking the two contrasts of the conditions of colour-certainty and shape-certainty against the rest condition with each other revealed mixed results for the colour sensitive areas V4 and V4 $\alpha$ (selectively higher activity in the left hemisphere during colour discriminations, nonselective activity in the right hemisphere during both certainty conditions) and relatively higher activity in shape sensitive area LOC during shape discriminations. The results of the differential contrasts between these two certainty conditions provide only little support for selective activity in V4 and no support for selective activity in the LOC under our conditions. There are different possible explanations for the fact that we did not find effects of the attended stimulus feature comparable to the earlier studies by Corbetta et al. (1990, 1991) and Le et al. (1998). One possibility is that the pronounced effects found by Le et al. (1998) are due to the large stimulus differences they used in their study and that the net effect of the direction of selective attention on the activity in specialised areas of extrastriate visual cortex is much smaller than premised. On the other hand, at least one methodological aspect might have diluted the effect of attention in our study. Changes in the baseline MR signal across the different scanning runs might have confounded the experimental effects since only one experimental condition was carried out per run. Presenting all experimental conditions within each run would have minimised the effect of these baseline shifts.

When the subjects' performances in the two certainty and the corresponding uncertainty conditions are compared, it is obvious that judgements are uniformly less accurate during uncertainty. This means that the sensitivity to near-threshold differences is much higher when subjects know in advance on which feature dimension a change will occur, so that they can direct their attention selectively to this feature, compared to when they have to divide up their attentional resources to different features of an object - an effect that has also been reported by Corbetta et al. $(1990,1991)$ and Thomas and Olzak (1996) among others. This effect can be explained by the fact that during stimulus uncertainty, decisions are perturbed by noise effects from two sources instead of only one source as during stimulus certainty (Tanner 1956; Thomas and Olzak 1996).

The results of the statistical analysis of the fMRI data indicate pronounced differences in cortical activity between certainty and uncertainty. Foci of higher cortical activity during the uncertainty condition mainly lie in lateral prefrontal (BAs 9, 10, 46), posterior parietal (BAs 7 and 40) and premotor areas (BA 6) that have been shown before to be essential for attentional and working memory processes (see Kanwisher and Wojciulik 2000; D'Esposito et al. 1998). As many different studies have demonstrated, lateral prefrontal and posterior parietal areas seem to be parts of a complex fronto-parietal attention network controlling attentional modulation of neural activity. It might be inferred that the higher degree of activation during the uncertainty condition in these areas goes back to the higher level of demand on attention and working memory during stimulus uncertainty. As shown before by several other studies, posterior parietal cortex is consistently activated during spatial as well as nonspatial visual search (Coull et al. 2003) independent of the presence of distractors (Donner et al. 2003). Furthermore, activity in the premotor and dorsolateral prefrontal cortex previously has been related to response conflict (e.g., Casey et al. 2000), which is also more pronounced during stimulus uncertainty than during certainty, since during uncertainty there are four stimulus alternatives to choose from instead of only two alternatives as during certainty. Another possible reason for higher activity in these cortical areas is the demand for frequent changes in cognitive set during the uncertainty condition. The switching between colour and shape discriminations is quite similar to other task switching paradigms, for which higher activity in these cortical areas has been demonstrated in previous studies (e.g., Brass et al. 2003; Sohn et al. 2000).

Taken together, the results of this study provide limited support for an effect of the direction of nonspatial selective visual attention on the activity of specialised areas of human extrastriate visual cortex. In addition to this finding, the crucial role of different lateral prefrontal and posterior parietal cortical areas for executive functions related to attention, working memory and changes in cognitive set has been confirmed by our uncertainty paradigm.

Acknowledgements This research was funded by the German Research Foundation (SFB 517, C9). We also thank the Hanse Institute for Advanced Study in Delmenhorst, Germany, for providing Prof. James P. Thomas with financial support. Additionally, we would like to thank Prof. Dr. Burckhard Terwey of the "Zentralkrankenhaus Sankt-Jürgen-Straße" in Bremen, Germany, who gave us access to his MR scanner for this experiment. Finally, we are grateful to reviewers for supporting us with helpful comments on an earlier version of this article. A preliminary account of these findings has been published in proceedings form (Weerda, Vallines, Thomas and Greenlee 2003). J. P. Thomas was a fellow of the Hanse Institute for Advanced Study, Delmenhorst, Germany. This study has been approved by the ethics committee of the Carl von Ossietzky University Oldenburg and has, therefore, been performed in accordance with the ethical standards of the 1964 Declaration of Helsinki.

\section{References}

Bartels A, Zeki S (2000) The architecture of the colour centre in the human visual brain: new results and a review. Eur J Neurosci $12: 172-193$

Blanco MJ, Soto D (2002) Effects of spatial attention on detection and identification of oriented lines. Acta Psychol (Amst) 109:195-212

Brass M, Ruge H, Meiran N, Rubin O, Koch I, Zysset S, Prinz W, von Cramon DY (2003) When the same response has different meanings: recoding the response meaning in the lateral prefrontal cortex. NeuroImage 20:1026-1031 
Casey BJ, Thomas KM, Welsh TF, Badgaiyan RD, Eccard CH, Jennings JR, Crone EA (2000) Dissociation of response conflict, attentional selection, and expectancy with functional magnetic resonance imaging. Proc Nat Acad Sci USA 97:8728-8733

CIE (1932) Commission Internationale de l'Eclairage Proceedings, 1931. Cambridge University Press, Cambridge

Corbetta M, Miezin F, Dobmeyer S, Shulman GL, Petersen SE (1990) Attentional modulation of neural processing of shape, color, and velocity in humans. Science 248:1556-1559

Corbetta M, Miezin F, Dobmeyer S, Shulman GL, Petersen SE (1991) Selective and divided attention during visual discriminations of shape, color, and speed: functional anatomy by positron emission tomography. J Neurosci 11:2383-2402

Coull JT, Walsh V, Frith CD, Nobre AC (2003) Distinct neural substrates for visual search amongst spatial versus temporal distractors. Cogn Brain Res 17:368-379

D'Esposito M, Aguirre GK, Zarahn E, Ballard D (1998) Functional MRI studies of spatial and non-spatial working memory. Cogn Brain Res 7:1-13

Donner TH, Kettermann A, Diesch E, Villringer A, Brandt SA (2003) Parietal activation during visual search in the absence of multiple distractors. NeuroReport 14:2257-2261

Friston KJ, Holmes AP, Worsley KJ, Poline JB, Frith CD, Frackowiak RS (1995) Statistical parametric maps in functional imaging: a general linear approach. Hum Brain Mapp 2:189210

Graham NVS (1989) Visual Pattern Analyzers. Oxford University Press, New York

Grill-Spector K, Kourtzi Z, Kanwisher N (2001) The lateral occipital complex and its role in object recognition. Vis Res 41:1409-1422

Huk AC, Heeger DJ (2000) Task-related modulation of visual cortex. J Neurophysiol 83:3525-3536

Ishihara S (1982) Ishihara's tests for colour blindness, 38 plates edition. Tokyo etc.: Kanehara

Kanwisher N, Wojciulik E (2000) Visual attention: insights from brain imaging. Nat Rev Neurosci 1:91-100

Kourtzi Z, Kanwisher N (2000) Cortical regions involved in perceiving object shape. J Neurosci 20:3310-3318

Kourtzi Z, Kanwisher N (2001) Representation of perceived object shape by the human lateral occipital complex. Science 293:1506-1509

Le TH, Pardo JV, Hu X (1998) 4 T-fMRI study of nonspatial shifting of selective attention: cerebellar and parietal contributions. J Neurophysiol 79:1535-1548

Lerner Y, Hendler T, Ben-Bashat D, Harel M, Malach R (2001) A hierarchical axis of object processing stages in the human visual cortex. Cereb Cortex 11:287-297
Magnussen S, Greenlee MW (1997) Competition and sharing of processing resources in visual discrimination. J Exp Psychol Hum Percept Perform 23:1603-1616

Malach R, Reppas JB, Benson RR, Kwong KK, Jiang H, Kennedy WA, Ledden PJ, Brady TJ, Rosen BR, Tootell RBH (1995) Object-related activity revealed by functional magnetic resonance imaging in human occipital cortex. Proc Nat Acad Sci USA 92: 8135-8139

Morgan MJ, Watamaniuk SNJ, McKee SP (2000) The use of an implicit standard for measuring discrimination thresholds. Vis Res 40:2341-2349

Pelli DG (1985) Uncertainty explains many aspects of visual contrast detection and discrimination. J Opt Soc Am A 2:1508-1532

Sohn M-H, Ursu S, Anderson JR, Stenger VA, Carter CS (2000) The role of prefrontal cortex and posterior parietal cortex in task switching. Proc Nat Acad Sci USA 97:13448-13453

Sperling G, Dosher BA (1986) Strategy and optimization in human information processing. In: Boff KR, Kaufman L, Thomas JP (eds) Handbook of perception and human performance, vol. I: Sensory processes and perception. Wiley, New York

Talairach J, Tournoux P (1988) Co-planar stereotaxic atlas of the human brain. Thieme, Stuttgart

Tanner WP (1956) Theory of recognition. J Acoust Soc Am 28:883-888

Thomas JP, Olzak LA (1996) Uncertainty experiments support the roles of second-order mechanisms in spatial frequency and orientation discriminations. J Opt Soc Am A 13:689-696

Thomas JP, Magnussen S, Greenlee MW (2000) What limits simultaneous discrimination accuracy? Vis Res 40:3169-3127

Treisman A (1986) Properties, part, and object. In: Boff KR, Kaufman,L, Thomas JP (Eds) Handbook of perception and human performance, Vol. II: Cognitive processes and performance. Wiley, New York

Vallines JI, Thomas JP, Greenlee MW (2001) Concurrent judgements of colour and shape. Perception 30 (suppl 14)

Vogels R, Eeckhout H, Orban GA (1988) The effect of feature uncertainty on spatial discriminations. Perception 17:565-577

Weidner R, Pollmann S, Müller HJ, von Cramon DY (2002) Topdown controlled visual dimension weighting: an event-related fMRI study. Cereb Cortex 12:318-328

Weerda R, Vallines I, Thomas JP, Greenlee MW (2003) Individual differences in cognitive strategies and attentional effects during stimulus uncertainty: evidence from psychophysics and fMRI. In: Reinvang I, Greenlee MW, Herrmann M (Eds) The cognitive neuroscience of individual differences - new perspectives. Hanse Studies, Vol 4. Bibliotheks- und Informationssystem der Universität Oldenburg, Oldenburg

Wickens TD (2001) Elementary signal detection theory. Oxford University Press, Oxford 